\title{
State of the science on mild cognitive impairment (MCI)
}

\author{
Nicole D. Anderson (iD) *
}

Departments of Psychology and Psychiatry, University of Toronto, Rotman Research Institute, Baycrest Health Sciences, Toronto, Ontario, Canada

Mild cognitive impairment (MCI) represents a transitional stage between healthy aging and dementia, and affects $10-15 \%$ of the population over the age of 65. The failure of drug trials in Alzheimer's disease (AD) treatment has shifted researchers' focus toward delaying progression from MCI to dementia, which would reduce the prevalence and costs of dementia profoundly. Diagnostic criteria for MCI increasingly emphasize the need for positive biomarkers to detect preclinical AD. The phenomenology of MCI comprises lower quality-of-life, greater symptoms of depression, and avoidant coping strategies including withdrawal from social engagement. Neurobiological features of MCI are hypoperfusion and hypometabolism in temporoparietal cortices, medial temporal lobe atrophy particularly in rhinal cortices, elevated tau and phosphorylated tau and decreased $\mathrm{A} \beta_{42}$ in cerebrospinal fluid, and brain $\mathrm{A} \beta_{42}$ deposition. Elevated tau can be identified in MCI, particularly in the entorhinal cortex, using positron emission tomography, and analysis of signal complexity using electroencephalography or magnetoencephalography holds promise as a biomarker. Assessment of MCI also relies on cognitive screening and neuropsychological assessment, but there is an urgent need for standardized cognitive tests to capitalize on recent discoveries in cognitive neuroscience that may lead to more sensitive measures of MCI. Cholinesterase inhibitors are frequently prescribed for MCI, despite the lack of evidence for their efficacy. Exercise and diet interventions hold promise for increasing reserve in MCI, and group psychoeducational programs teaching practical memory strategies appear effective. More work is needed to better understand the phenomenology and neurobiology of MCI, and how best to assess it and delay progression to dementia.

Received 6 February 2018; Accepted 28 August 2018; First published online 17 January 2019

Key words: Amyloid, biomarkers, exercise and diet interventions, memory strategy training, mild cognitive impairment, neuroimaging, neuropsychological assessment, tau.

\section{Introduction}

A new case of dementia is diagnosed every 3 seconds, the equivalent of 9.9 million new cases globally in $2015 .^{1}$ The worldwide prevalence of dementia is expected to almost double every 20 years, from 46.8 million in 2015 to 131.5 million in 2050, with most of the increase occurring in low and middle income countries. ${ }^{1}$ The combined cost of health care and loss of earnings due to dementia, already at $\$ 81$ billion (USD) per year, is predicted to rise to $\$ 2$ trillion by 2030. ${ }^{1}$ Considering Alzheimer's disease (AD) in particular, no disease-modifying treatment exists, and clinical drug trials in the past decade have had a $99.6 \%$ failure rate. ${ }^{2,3}$ One contributor to this high failure rate is the fact that brain pathology begins years before onset of objective cognitive symptoms and may be irreversible by the time of diagnosis. ${ }^{4}$ These depressing statistics have led many investigators to

* Address for correspondence: Nicole D. Anderson, PhD, CPsych, Associate Professor, Departments of Psychology \& Psychiatry, University of Toronto, Senior Scientist, Rotman Research Institute, Baycrest Health Sciences, 3560 Bathurst Street, Toronto, ON, Canada M6A 2 E1.

(Email: nanderson@research.baycrest.org) switch their focus toward delaying dementia in persons who are in preclinical phases of the disease. Economic analyses have concluded that a treatment introduced in 2025 that delays the onset of dementia by five years would reduce the prevalence and associated healthcare costs of dementia by approximately $40 \%$ over the following 25 years. $^{5}$

Mild cognitive impairment (MCI) represents the preclinical, transitional stage between healthy aging and dementia and represents what researchers and clinicians view as a "window" in which it may be possible to intervene and delay progression to dementia. The goal of this article is to broadly cover the state of the current science on MCI, including its phenomenology and neurobiology, how MCI is screened for and assessed, and interventions designed to improve cognitive functioning in MCI, thereby delaying progression to dementia. To set the stage for these discussions, this article begins by describing the evolving operational definitions of MCI and its prevalence.

The notion of preclinical cognitive decline has existed for decades, and was variously termed, eg, "aging-associated cognitive decline"6 and "cognitive impairment, no dementia."7 
Another important conceptualization is the Clinical Dementia Rating (CDR) of 0.5, termed "questionable dementia." A CDR of 0.5 includes the following features, as judged by a clinician: (1) mild consistent forgetfulness; partial recollection of events; (2) fully oriented; (3) only doubtful or mild impairment, if any, in independent functioning; (4) no major influence on home life, hobbies, or intellectual interests; and (5) fully capable of basic activities of daily living. Researchers at Washington University in St. Louis, Missouri, in particular have advocated the use of the CDR, arguing that what others call MCI is in fact early- $\mathrm{AD}$, and therefore there is no need to view MCI (or a CDR of 0.5 ) as a separate entity from $\mathrm{AD}{ }^{9}$

The term MCI was introduced in $1988,{ }^{10}$ but became common nomenclature when Ron Petersen and his colleagues at the Mayo Clinic in Rochester, Minnesota, developed the first clinical criteria for MCI in $1999 .{ }^{11}$ The original Mayo criteria were the following: (1) memory complaint, preferably corroborated by an informant; (2) objective memory impairment for age and education; (3) largely normal general cognitive function; (4) essentially normal activities of daily living; and (5) not demented. Thus, the focus was very much on what is now called amnestic MCI, given the key criterion that memory, of all cognitive domains, must be impaired. Later, the Mayo group suggested different subtypes of MCI, ${ }^{12,13}$ representing the factorial crossing of singledomain/multiple-domain and amnestic/nonamnestic. For example, someone with deficits in memory and 1 or more other cognitive domain (eg, language, executive functioning, visuospatial ability) would have multipledomain amnestic MCI. Someone with solely an executive functioning impairment would have single-domain nonamnestic MCI, and is likely to be in the preclinical stages of a dementia other than $\mathrm{AD}$ (eg, frontotemporal dementia or vascular dementia).

The tide has swung even further away from memory impairment as a key feature of preclinical AD MCI. The recent guidelines put forth by the National Institute on Aging-Alzheimer's Association (NIA-AA) workgroups distinguished between clinical criteria and research recommendations for "MCI due to $\mathrm{AD} .{ }^{14}$ The clinical criteria are the following: (1) concern regarding a change in cognition, from the patient, an informant, or a skilled clinician; (2) impairment in one or more cognitive domain relative to a person's age and educational attainment; (3) independence in functional abilities (although they make take more time or be performed less efficiently); and, (4) not demented. Thus, the main difference between the original and the newer clinical criteria is that individuals do not have to have memory impairment to meet criteria for MCI due to $\mathrm{AD}$. The clinical diagnosis of MCI, these newer guidelines also state, should also involve a clinical evaluation to confirm a likely $\mathrm{AD}$ etiology of the individual's cognitive impairment (eg, family history of $\mathrm{AD}$, ruling out other possible causes). Separate research recommendations for the diagnosis of MCI due to $\mathrm{AD}$ include biomarkers of $\mathrm{A} \beta$ deposition and neuronal injury: individuals with positive tests of both biomarkers are considered to be highly likely to have MCI due to $\mathrm{AD}$, individuals with positive tests on one biomarker are considered to have an intermediate likelihood of having MCI due to $\mathrm{AD}$, and individuals for whom both biomarkers are negative are considered to have MCI, but unlikely due to AD.

Finally, in 2013, the American Psychiatric Association, in its Diagnostic and Statistical Manual for Mental Disorders, Fifth Edition, ${ }^{15}$ developed the term mild neurocognitive disorder (mild NCD) to describe MCI. The clinical criteria for mild NCD include: (1) evidence of cognitive decline in one or more cognitive domains, obtained from reports from the client, an informant, or a clinician, or from objective testing; (2) preserved functional independence; (3) the cognitive impairments do not occur exclusively during episodes of delirium; (4) the cognitive deficits cannot be better explained by another condition (eg, depression); and (5) no dementia. These criteria, unlike those of the NIA-AA, are not specific to MCI due to preclinical $\mathrm{AD}$, but rather include a second diagnostic step of determining the potential etiology of the cognitive deficits (eg, $\mathrm{AD}$, frontotemporal dementia, vascular disease, traumatic brain injury, substance abuse). Despite these being the most recent criteria, the term MCI still dominates in research and clinical circles.

Prevalence estimates of MCI vary widely, with one review reporting prevalence as low as $3 \%$ to as high as $42 \%$ across 35 studies, varying across country of study origin, and as a function of the age range of study inclusion, with lower prevalence in studies that included younger participants. ${ }^{16,17}$ Prevalence of MCI has also been reported to be higher in clinic-based than community-based samples. ${ }^{18,19}$ Approximately 10-15\% of people with amnestic MCI progress to $\mathrm{AD}$ each year, compared to $1-2 \%$ of the healthy older adult population. ${ }^{11,20}$ It is thus imperative that we understand the phenomenology, neurobiology, and clinical characteristics of MCI, and how best to treat it, in efforts to improve functioning and delay progression to dementia.

\section{Phenomenology of MCI}

The phenomenology of MCI has been examined using both quantitative and qualitative research methods. Studies using a quantitative approach, such as administering questionnaires to people with MCI and healthy older adults, have found reduced quality-of-life in individuals with MCI, ${ }^{21,22}$ particularly when they are aware of their diagnosis. ${ }^{23}$ Symptoms of depression, anxiety, hostility, and low morale have also been reported to be elevated in people with MCI compared to healthy 
older adults. ${ }^{24}$ Indeed, people with MCI experience depressive symptoms at approximately double the rate of healthy seniors, ${ }^{25,26}$ with one of these studies finding that symptoms of depression, including those that met the cutoff for clinical significance, were elevated in individuals with amnestic, but not non-amnestic, MCI. ${ }^{26}$ The authors argued that depression can result when memory difficulties interfere with the ability to perform instrumental activities of daily living, but it should be noted that such activities also rely on executive functions. ${ }^{27}$

A handful of qualitative studies have shared the lived experience of people with mild cognitive impairment. Roberts and Clare ${ }^{28}$ used interpretative phenomenological analysis of interview data with 25 people who had been diagnosed with MCI, a method which interprets the meaning of the statements shared by participants. Their analysis identified 4 themes. The first theme reflected participants' appraisals of their cognition in the context of their perceived social network: individuals living alone identified retention of independence as being of high importance, in fear it might be taken away, while those who lived with others leaned on their partners to maintain their independence. The second theme reflected a normalization of participants' own memory problems, viewing them as a normal consequence of aging, with comments such as "All my friends forget things too." The third theme described participants' unwillingness to consider negative impact or outcomes as a result of their diagnoses. Participants often used humor to deflect the seriousness of their condition. The final theme reflected participants' lack of sophisticated knowledge about what MCI is and their concerns for the future. While these last two themes may seem to contradict each other, the frequent vacillation between memory concerns and minimization of the significance of their diagnosis is commonly observed in clinical practice, often times even in a single statement, eg, "I worry all the time I might become demented, but then again, all my friends forget things!"

Berg et $a l^{29}$ interviewed 17 individuals who maintained a diagnosis of MCI across 4 assessments over a 7year period. The majority of them expressed wanting to spare their children of the experiences they had caring for their mothers with dementia. Another common theme, like the results of Roberts and Clare, ${ }^{28}$ was attribution of the experienced memory difficulties to normal aging, a minimization which may also be a form of avoidant coping. Most participants expressed concerns for the future and the impending loss of capabilities and the impact that this will have on their participation in activities they find meaningful. Finally, participants reported avoidant coping behaviors, reducing stress by avoiding situations they thought they could not handle.

Avoidant coping was also a key observation of the qualitative study of 14 individuals with amnestic MCI reported by Parikh et al. ${ }^{30}$ These individuals reported withdrawing from the social activities they previously enjoyed to avoid embarrassment and frustration when their memory fails them in these settings. Social exclusion was also reported, such as being interrupted when they hesitate in conversations, and feeling that their friends no longer share information with them in the way they had previously.

In summary, this literature paints a picture of reduced quality of life, elevated symptoms of depression, and avoidant coping strategies such as minimizing the significance of one's diagnosis and withdrawing from leisure activities as key features of the phenomenology of MCI. Treating depression and not only encouraging, but facilitating, social engagement are important elements in the treatment of MCI, as discussed in the final section of this article, particularly given the identified role of depression and reduced social engagement as risk factors for dementia ${ }^{31,32}$ and the influence of depression in the progression from MCI to dementia. ${ }^{33}$

\section{Neurobiology of MCI}

All of the neuropathologies identified in MCI cohorts can also be found in seniors with intact cognition and in seniors with dementia, and typically fall in the intermediate stage in terms of severity. ${ }^{34}$ These include atrophy of medial temporal lobe regions, particularly the hippocampus and entorhinal regions, and posterior cingulate cortex, ${ }^{35}$ hypometabolism in temporoparietal and posterior cingulate cortices, as assessed by fluorodeoxyglucose positron emission tomography (FDGPET) ${ }^{36}$ and hypoperfusion of parietal cortices and the hippocampus as measured by single-photon emission computed tomography (SPECT). ${ }^{37}$

The other primary neurobiological markers of MCI pertain to the neuropathology of $\mathrm{AD}$-amyloid plaques and neurofibrillary tangles. Evidence of amyloid accumulation in MCI (and AD) can be detected with positron emission tomography using the (11)C-labelled Pittsburgh Compound-B ((11)C-PIB) ligand. The great variability in how PIB scans are performed and interpreted has raised calls for greater standardization of this high cost procedure. ${ }^{38}$ Amyloid beta $\left(\mathrm{A} \beta_{42}\right)$ can be detected in the cerebrospinal fluid (CSF) at much lower cost, with lower CSF $A \beta_{42}$ concentrations indicating higher levels of brain $\mathrm{A} \beta_{42}$ (ie, poorer clearance), whereas elevated CSF concentrations of total tau and phosphorylated tau indicate neuronal injury and predict progression from MCI to AD-related dementia. Both CSF $\mathrm{A} \beta_{42}$ and tau, as well as PET PIB, have better sensitivity than specificity, ${ }^{39,40}$ suggesting that they are more useful in ruling out preclinical $\mathrm{AD}$ than in confirming it.

The epsilon 4 allele of the apolipoprotein gene (APOE-e4) confers a risk for MCI, as it does for $\mathrm{AD} .^{41}$ Differences in gene expression in cortical brain regions 
have been reported in MCI that are unique compared to healthy cohorts and those with $\mathrm{AD} .^{42}$ These authors report an upregulation of genes associated with anabolic and biosynthetic pathways and mitochondrial energy generation. Upregulation of genes associated with synaptic transmission and plasticity was also identified in entorhinal cortex and hippocampus, but this was associated with poorer cognitive functioning as assessed with the Mini-Mental State Examination (MMSE). ${ }^{43}$ This finding is reminiscent of previous reports of a "paradoxical" hyperactivation of the hippocampus during encoding in MCI, with greater hyperactivation associated with greater atrophy and cortical thinning in medial temporal lobe regions. ${ }^{44,45}$ One interpretation of this apparent paradox is that it is the brain's last ditch attempt to maintain functioning in the face of mounting neuropathology.

The elevated rates of depression in MCI deserve mentioning in the context of the neurobiology of MCI. There is much debate about the causal relationship between depression and MCI. As spelled out by Shahnawaz et al, ${ }^{26} 4$ mechanisms of this relationship have been proposed: (1) the downstream consequences of depression (eg, elevated cortisol and its effects on the brain) may cause cognitive decline $;^{46}(2)$ depression may be an early outcome of MCI as individuals experience cognitive decline $;^{47}(3)$ an independent, third variable commonly comorbid with both conditions, such as vascular disease, may be causing both depression and cognitive decline ${ }^{48}$ and (4) medications for depression may exacerbate cognitive decline due to their anticholinergic effects. ${ }^{49}$

Emerging approaches in neuroimaging may prove useful for early detection of MCI. For example, altered synchronization of brain signals has been reported using magnetoencephalography (MEG) ${ }^{50}$ and functional magnetic resonance imaging (fMRI). ${ }^{51}$ Likewise, electroencephalography (EEG) and MEG studies have shown increased power in low frequencies (delta and theta bands) and decreased power in higher frequencies (alpha and beta bands) in people with MCI compared to controls, ${ }^{52}$ suggesting a shift away from firing patterns required for complex cognitive processes, such as working memory and avoiding distraction, toward more basic processes such as orienting attention. Analyses of the complexity of EEG and MEG time-series data, such as entropy, typically show reduced signal complexity compared to healthy older adults, ${ }^{52,53}$ suggesting that people with MCI have less complex (ie, more regular) neural dynamics, thus limiting the repertoire of brain states and restricting transitions between states. ${ }^{54}$ Moreover, the synchrony of resting-state EEG oscillatory neural activity may be reduced in $\mathrm{MCI},{ }^{55}$ restricting short-range and long-range communication.

A final neurobiological biomarker that is also not part of standard diagnostic practices, but has promise for the future, is the structural and functional integrity of perirhinal cortex. Given that Alzheimer's pathology begins in the transentorhinal cortex, ${ }^{56}$ which corresponds to the medial perirhinal cortex, ${ }^{57}$ it is not surprising that a hallmark feature of amnestic MCI is atrophy and thinning of the rhinal cortices (see Zhou et $a l^{58}$ for a review), combined with hippocampal atrophy that exceeds that seen in healthy aging (see Leal and Yassa $^{59}$ for a review). The volume of the anterolateral entorhinal cortex has recently been reported to correlate with performance on the Montreal Cognitive Assessment (MoCA), ${ }^{60}$ a cognitive screening tool that is sensitive to MCI, in a group of healthy older adults, some of whom had failed the MoCA. ${ }^{61}$ Perirhinal neurofibrillary tangle deposition in people with MCI has also been recently reported, ${ }^{62}$ and in early $\mathrm{AD}$, greater perirhinal atrophy is associated with greater tau deposition, whereas greater hippocampal atrophy is associated with greater amyloid deposition. ${ }^{63}$ Thus, the deployment of high-resolution structural magnetic resonance imaging to assess the volume and cortical thickness of perirhinal cortices, and the combination of fMRI with clever tasks that drive perirhinal and lateral entorhinal processes, as discussed in the next section of this article, may be fruitful avenues for early detection of MCI.

\section{Assessment of $\mathrm{MCl}$}

The current clinical and research criteria for diagnosis of MCI were enumerated at the outset of this article. This section will comment on how those criteria are assessed. In clinical practice, and as per the research criteria for the assessment of MCI, neuroimaging is often performed. This can include SPECT and/or FDG-PET to verify hypoperfusion and glucose hypometabolism of temporoparietal cortices, respectively; structural MRI to identify medial temporal lobe atrophy; and CSF assays to verify elevated levels of tau and phosphorylated tau, all indicative of neuronal injury. Likewise, reduced CSF levels of $A \beta_{42}$ (indicative of elevated brain levels of $A \beta_{42}$ ) or of $A \beta_{42}$ deposition in the brain using PET imaging are increasingly common tests at least in better resourced communities to confirm MCI diagnosis. A recent international working group published guidelines for CSF biomarker in the diagnosis of MCI concluded that CSF biomarkers hold significant utility in predicting conversion to $\mathrm{AD} 3$ years later, but the evidence was too limited to recommend any one CSF biomarker over another. ${ }^{64}$ Finally, confirmation that a client carries 1 or $2 \varepsilon 4$ alleles in the APOE gene increases confidence in the diagnosis of MCI due to $\mathrm{AD}$.

Researchers are constantly aiming to identify lower cost, less invasive biomarkers of $\mathrm{AD}$ neuropathology in the preclinical phases of the disease. One group recently reported the utility of a blood plasma-based composite 
biomarker of $\mathrm{A} \beta$ deposition that fared very well against PIB-PET imaging and CSF A $\beta{ }^{65}$ Thus, the notion that individuals can receive an early diagnosis in their family physician's office, and then be directed toward treatments that delay progression to dementia, is foreseeable.

In addressing the diagnostic criteria of cognitive impairment in clinical practice, it is not uncommon for physicians to rely solely on a cognitive screening measure such as the MMSE or the MoCA. The MMSE, however, lacks in sensitivity to detect MCI, ${ }^{60}$ and the MoCA is insufficient to determine the specific cognitive domains affected in order to determine MCI subtype (although see Julayanont et $a l^{66}$ for an approach that hopefully will be validated against a more extensive assessment). There are 3 versions of the MoCA, permitting re-assessment without undue influence of practice effects; however, the first version of the test was published by media outlets in January 2018, following the news that President Trump had passed it, and so clinicians are now recommended to avoid using this version in case their clients have become familiar with the test items.

A more comprehensive approach to cognitive assessment involves a neuropsychological assessment. Neuropsychologists typically administer a 90-minute to 3-hour assessment of cognitive functioning and factors that could influence performance (eg, mood, self-report of learning disabilities, other major medical conditions, sensory and language fluency limitations). The neuropsychological assessment typically includes measures of memory, executive functioning, attention, language, and visuospatial skills. In the diagnosis of MCI (and other conditions for that matter), it is important to find evidence of impairments across multiple tests within a cognitive domain, such as on multiple tests of episodic memory. This is because a surprisingly high percentage of healthy seniors will score below cut-off on at least 1 memory measure in a battery $(\mathrm{eg}, 38 \%$ scoring $<5$ th percentile in Brooks et at $t^{67}$ and $>60 \%$ scoring $<10$ th percentile in Mistridis et $\left.a l^{68}\right)$, rendering the potential for misdiagnosis of MCI high if made on the basis of 1 failed test. Those examples also highlight the variability in the literature and in clinical practice in how a cognitive "impairment" is operationalized, with some using a cut-off of 1.5 standard deviations (SD) below the age- (and education-, where available) corrected normative data $(<7$ th percentile), and others using a less stringent -1.0 SD cut-off (<16th percentile). This inconsistency in diagnostic practice contributes to the variability in not only prevalence and incidence rates of MCI, but also in its prognostic patterns.

Failing to take a clients' medical and psychosocial history into account in differential diagnosis can likewise lead to misclassification of MCI; for example, if a client reports that his memory difficulties emerged following open-heart surgery, but have not continued to decline, memory impairment secondary to hippocampal damage due to anoxia could be mistaken for preclinical dementia. Issues such as these likely contribute to the sometimes high rates of "reversion to normal" when people previously diagnosed with MCI are tested later and score within normal ranges (for a meta-analysis, see MalekAhmadi ${ }^{69}$ ).

Another contentious aspect about the assessment of MCI has been the criterion regarding subjective concern. The unreliable nature of memory concerns in MCI that was mentioned in a previous section of this article has been validated by studies reporting poor associations between the degree of subjective memory concerns and actual memory performance in $\mathrm{MCI}^{70}$ (see Roberts et $a l^{71}$ for an earlier review), most typically reflecting an underestimation of the magnitude of actual impairment. ${ }^{72}$ This has led many investigators to question the reliability of the patients' concerns about their memory (versus the concerns of a family member and/or healthcare professional) as a core clinical criterion for MCI. Yet, it should be noted that the impetus for including in the diagnostic criteria a concern that one's memory (or other cognitive domain) is becoming worse was to distinguish a probable neurodegenerative process from a non-degenerative process.

A final point about the cognitive assessment of MCI relates to the assessment tools used in neuropsychology, which have a rich history, with most developed or at least rooted in early efforts to understand brain-behavior relationships. New versions of tests are frequently released, but the tests remain essentially the same. One implication of the relative rigidity of neuropsychological practices is that assessment tends not to capitalize on recent advances in our understanding of the neurobiology of conditions, including MCI. Given that the earliest neuropathological changes in MCI occur in the perirhinal cortex, as mentioned in the previous section of this article, cognitive tests that tap into perirhinal functioning may prove to be particularly sensitive to MCI. The perirhinal cortex and lateral entorhinal cortex have been discovered to mediate complex object discrimination, ${ }^{73-75}$ whereas spatial information is processed by an alternate medial temporal lobe route involving the parahippocampal gyrus and medial entorhinal cortex. ${ }^{76}$ Indeed, compared to their healthy counterparts, people with aMCI report problems with "object memory," forgetting what things are called, what they watched on television, to do things that are right in front of them, but share similar complaints with healthy seniors about "spatial memory," such as where they put things or recognizing places. ${ }^{77,78}$ The perirhinal cortex has also been implicated in the mnemonic process of familiarity-the gut sense that you have experienced something before but you fail to remember the original context of the event. ${ }^{79-81}$ 
This literature would suggest that tests of complex object discrimination (and memory for objects when they have to be distinguished from highly similar lures) and tests of familiarity may be particularly sensitive to MCI. Indeed, MoCA performance was more strongly associated with the ability to select a studied item over a highly similar lure item for objects than scenes in the study by Fidalgo et $a l,{ }^{82}$ and people with MCI have greater difficulty identifying objects when they are presented in an overlapping line-drawn display. ${ }^{83}$ The literature on whether familiarity is spared or impaired is split, with approximately half of the studies finding spared familiarity in MCI $\left(\mathrm{eg},{ }^{84,85}\right)$ and the other half finding familiarity deficits in MCI $\left(\mathrm{eg},{ }^{86,87}\right)$. This author believes this confusion can be attributed to how familiarity is typically assessed experimentally, pitting it against recollection (the ability to recall a prior event and its context), and advocates for more novel approaches to assess familiarity free from the influence of recollection. Indeed, one study in this vein had older adults who were either carriers or noncarriers of APOE $\varepsilon 4$ study faces against a red or blue background. ${ }^{88}$ Later, they performed a yes/no recognition task of the studied faces intermixed with new faces, and then for faces deemed "old" (studied), indicated if it was studied with a red or blue background. The two groups did not differ in the ability to recognize a studied face and remember its context (recollection), but $\varepsilon 4$ carriers were less likely to recognize a studied face but not remember its studied context (familiarity).

Despite all of the neurobiological reasons to think that complex object discrimination or familiarity may be exquisitely sensitive to MCI, standardized tests of these abilities have not been developed. It is high time that the assessment of MCI (and other neurological disorders) catches up with state of neuroscience research findings.

\section{Treatment of MCI}

Given that MCI, particularly in its amnestic form, is often a precursor to $\mathrm{AD}$, it is not surprising that most (70\%) physicians report prescribing cholinesterase inhibitors "off-label" at least sometimes in this cohort, and $39 \%$ reported prescribing memantine. ${ }^{89}$ These prescribing practices occur despite the continued lack of high-quality evidence for their efficacy in $\mathrm{MCI},{ }^{16}$ perhaps because physicians suspect that their patients might have progressed to the early stages of $\mathrm{AD} .{ }^{89}$ Likewise, there is insufficient evidence to support treatment of MCI with alternate agents such as the nicotine patch or vitamin E. ${ }^{16}$ Physicians are recommended to wean their patients off other medications that may impair cognition, and to treat other modifiable risk factors that may be contributing to cognitive impairment, including mood disorders. $^{16}$
Given that there are no disease-modifying agents for MCI, and that the symptomatic treatments for AD lack efficacy in MCI, what can be done for people in this precarious state? The approaches described in the remainder of this section are not cures, and no one holds the belief that these measures will have direct effects on the neuropathological changes occurring in MCI, but there is still reason to think that they may delay progression to dementia. This thought rests on the fact that to progress to dementia one has to have cognitive deficits that are severe enough to interfere significantly with everyday functioning. Thus, if we can raise an individual's level of cognitive functioning, that person will have to experience a greater degree of cognitive decline before progressing to dementia, which will essentially buy him or her more time in relatively good cognitive health. This idea is a corollary of the notion of reserve $^{90}$ that has been offered to explain the discrepancy between the degree of neuropathology and one's level of cognitive functioning. Research has identified several early-life and mid-life factors, such as educational and occupational attainment, that increase reserve and permit individuals to tolerate higher levels of neuropathology yet maintain their cognitive functioning. ${ }^{90}$ Reserve can be neural, for example by fostering richer neural connections, or cognitive, allowing individuals to approach tasks in new ways. ${ }^{90}$ The approaches described below are efforts to enhance neural and/or cognitive reserve.

Physical exercise interventions have shown small but significant neural and cognitive benefits for cognition in people with MCI (for a meta-analysis, see Ströhle et a $l^{91}$ ). For example, Baker et a $t^{92}$ randomized 33 people with amnestic MCI to either aerobic training or a stretching control group 4 times a week over 6 months. In addition to the expected effects on cardiorespiratory fitness, aerobic training was associated with improved attention and executive functioning. However, the association between improvements in cardiorespiratory functioning $\left(\mathrm{VO}_{2}\right.$ peak) and these cognitive measures was larger for women than men. Aerobic exercise also had greater benefits for glucoregulatory function and plasma brain derived neurotrophic factor in women than men. These sex-specific improvements occurred despite the fact that both groups enjoyed comparable improvements in cardiorespiratory fitness. Similar reports of greater benefits of exercise, and physical activity more generally, in women than men have been reported in other metaanalytic and epidemiological studies ${ }^{93,94}$ and may be explained by the fact that the women in these studies tend to be less fit overall (body fat, lipid profiles, cortisol levels) at baseline, and thus have more room for improvement.

Adherence to Mediterranean-style dietary patterns has been associated with a reduced risk of MCI and $\mathrm{AD}$ in 
epidemiological studies. ${ }^{95}$ Few diet intervention studies in MCI populations have been published to date, but so far their results are promising. For example, BayerCarter $e t a l^{96}$ randomized 49 healthy older adults and 29 adults with amnestic MCI to either a high-saturated fat/high glycemic index (HIGH) diet or a low-saturated fat/low glycemic index (LOW) diet for four weeks. In the MCI group, compared to the HIGH diet, the LOW diet resulted in increased CSF A $\beta$ levels (indicative of improved brain clearance); increased CSF insulin concentrations; and decreased plasma lipids, insulin, and CSF F2-isoprostane concentrations. The beneficial effects of the LOW diet on CSF A $\beta$ were later found to be potentiated in individuals who reported engaging in more high intensity physical activity, ${ }^{97}$ hinting at the likely additive or synergistic effects of multicomponent approaches to healthy lifestyle interventions to increase reserve in people with MCI.

Another approach to increase reserve and everyday functioning in people with MCI is group psychosocial education. These interventions typically teach people practical, everyday memory strategies such as mental imagery and semantic processing to improve encoding of information; teach people about the effects of lifestyle factors such as exercise, diet, and cognitive and social engagement on memory and facilitate adoption of healthier lifestyles; and address coping strategies such as relaxation and preparing for the future. ${ }^{98-102}$ These studies have reported some improvements on objective tests of memory and other cognitive abilities ${ }^{98-101}$ and improvements in subjective memory, ${ }^{98,99,102}$ but due to variations in the quality of these studies (eg, use of an active control group, selection of appropriate outcome measures), the most recent MCI guidelines suggest that clinicians may (versus should) recommend such programs. ${ }^{16}$ More large-scale trials are needed with proper controls to identify the most effective group intervention approaches for MCI.

The MCI guidelines ${ }^{16}$ also highlight the need to educate patients and families about MCI, and suggest that clinicians should counsel patients and families. This type of counseling is common in some of these programs $\left(\mathrm{eg},{ }^{102}\right)$, but in response to an identified need for more, accessible information, the current author and her colleagues wrote a book in lay language about what MCI is, how it is diagnosed, and how it can be managed. ${ }^{103}$

In addition to these more comprehensive intervention approaches, a number of investigators are discovering the best approaches to memory interventions for MCI. For example, it has been repeatedly shown that people with MCI learn new information best if they are prevented from making errors while learning. ${ }^{104-106}$ Efforts to train working memory using adaptive approaches, meaning that the task becomes more difficult as individuals improve on the task, have shown improvements in working memory, but limited generalization to other cognitive domains. ${ }^{107,108}$ Likewise, trials of memory recollection training have shown limited generalization. ${ }^{109,110}$ These latter efforts indicate that restorative approaches, attempting to retrain specific cognitive processes, may be less effective cognitive rehabilitation approaches than compensatory approaches such as memory strategy training.

\section{Conclusions}

A massive amount of research has focused on honing the clinical criteria for MCI, and on gaining a better understanding of its prevalence and prognosis, its phenomenology and neurobiology, and how best to assess and treat MCI in efforts to delay progression to dementia. In fact, the number of articles that have been indexed in PubMed that can be found with the search term "mild cognitive impairment" since the term was introduced by Reisberg et $a l^{10}$ until the end of 2016 exceeds 12,000 , the vast majority of which (>94\%) have appeared since the beginning of the 21 st century. ${ }^{111}$ Yet, consensus still needs to be achieved concerning the clinical (and research) criteria for MCI and how to operationalize those criteria, and we still have a long way to go to better understand what it is like to live with MCI. As neuroimaging, genetic (and epigenetic), and molecular techniques advance, we will certainly learn much more about how MCI manifests in the brain. Academic neuropsychologists look forward to the day when standardized assessment tools catch up to the state of the neuroscience to develop new, validated behavioral or neurofunctional tests that prove more sensitive to the very early stages of MCI. And we all anticipate new treatments, pharmacological or otherwise, that improve prognosis of MCI and reduce prevalence of dementia.

\section{Disclosures}

Nicole Anderson receives royalties from Oxford University Press.

\section{REFERENCES:}

1. Alzheimer Disease International. World Alzheimer Report 2015: The Global Impact of Dementia. London, England. Retrieved from www.alz.co.uk. Accessed December 15, 2017.

2. Cummings J, Morstorf T, Zhong K Alzheimer's disease drugdevelopment pipeline: few candidates, frequent failures. Alzheimers Res Ther. 2014; 6(4): 37.

3. Schneider LS, Mangialasche F, Andreasen N, et al. Clinical trials and late-stage drug development for Alzheimer's disease: an appraisal from 1984 to 2014. J Intern Med. 2014; 275(3): 251-283. 
4. Sperling RA, Karlawish J, Johnson KA. Preclinical Alzheimer disease: the challenges ahead. Nat Rev Neurology. 2013; 9(1): 54-58.

5. Alzheimer's Association. Changing the trajectory of Alzheimer's disease: How a treatment by 2025 saves lives and dollars. Retrieved from www.alz.org. Accessed December 15, 2017.

6. Levy R Aging-associated cognitive decline. Int Psychogeriatr. 1994; 6(1): 63-68.

7. Graham JE, Rockwood K, Beattie BL, et al. Prevalence and severity of cognitive impairment with and without dementia in an elderly population. Lancet. 1997; 349(9068): 1793-1796.

8. Hughes CP, Berg L, Danzinger WL, Coben LA, Martin R. A new clinical scale for the staging of dementia. Br J Psychiatry. 1982; 140(6): 566-572.

9. Morris JC, Storandt M, Miller JP, et al. Mild cognitive impairment represents early-stage Alzheimer disease. Arch Neurol. 2001; 58 (3): 397-405.

10. Reisberg B, Ferris SH, de Leon MJ, et al. Stage-specific behavioral, cognitive, and in vivo changes in community residing subjects with age-associated memory impairment and primary degenerative dementia of the Alzheimer type. Drug Dev Res. 1988; 15(2-3): 101-114.

11. Petersen RC, Smith GE, Waring SC, Ivnik RJ, Tangalos EG, Kokmen E. Mild cognitive impairment: clinical characterization and outcome. Arch Neurol. 1999; 56(3): 303-308.

12. Petersen RC, Doody R, Kurz A, et al. Current concepts in mild cognitive impairment. Arch Neurol. 2001; 58(12): 1985-1992.

13. Petersen RC. Mild cognitive impairment as a diagnostic entity. J Intern Med. 2004; 256(3): 183-194.

14. Albert MS, DeKosky ST, Dickson D, et al. The diagnosis of mild cognitive impairment due to Alzheimer's disease: recommendations from the National Institute of AgingAlzheimer's Association working groups on diagnostic guidelines for Alzheimer's disease. Alzheimers Dement. 2011; 7(3): 270-209.

15. American Psychiatric Association. Diagnostic and Statistical Manual of Mental Disorders. 5th ed. Washington, DC: American Psychiatric Association; 2013.

16. Petersen RC, Lopez O, Armstrong MJ, et al. Practice guideline update summary: mild cognitive impairment. Neurology. 2018; 90 (3): 126-135.

17. Ward A, Arrighi HM, Michels S, Cedarbaum JM. Mild cognitive impairment: disparity of incidence and prevalence estimates. Alzheimers Dement. 2012; 8(1): 14-21.

18. Petersen RC, Caracciolo B, Brayne C, Gauthier S, Jelic V, Fratiglioni L. Mild cognitive impairment: a concept in evolution. J Intern Med. 2014; 275(3): 214-228.

19 Roberts R, Knopman DS. Classification and epidemiology of MCI. Clin Geriatr Med. 2013; 29(4), 753-772.

20. Farias ST, Mungas D, Reed BR, Harvey D, DeCarli C. Progression of mild cognitive impairment to dementia in clinic- vs communitybased cohorts. Arch Neurol. 2009; 66(9): 1151-1157.

21. Bárrios H, Narciso S, Guerreiro M, Maroco J, Logsdon R, de Mendonça A. Quality of life in patients with mild cognitive impairment. Aging Ment Health. 2013; 17(3): 287-292.

22. Teng E, Tassniyom K, Lu PH. Reduced quality-of-life ratings in mild cognitive impairment: analysis of subject and informant responses. Am J Geriatr Psychiatry. 2012; 20(12): 1016-1025.

23. Stites SD, Karlawish J, Harkins K, Rubright JD, Wolk D. Awareness of mild cognitive impairment and mild Alzheimer's disease dementia diagnoses associated with lower self-ratings of quality of life in older adults. J Gerontol B Psychol Sci Soc Sci. 2017; 72(6): 974-985.

24. Clément F, Belleville S, Bélanger S, Chassé V. Personality and psychological health in persons with mild cognitive impairment. Can J Aging. 2009; 28(2): 147-156.
25. Ravaglia G, Forti P, Lucicesare A, et al. Prevalent depressive symptoms as a risk factor for conversion to mild cognitive impairment in an elderly Italian cohort. Am J Geriatr Psychiatry. 2008; 16(10): 834-843.

26. Shahnawaz Z, Reppermund S, Brodaty H, et al. Prevalence and characteristics of depression in mild cognitive impairment: the Sydney Memory and Ageing study. Acta Psychiatr Scand. 2013; 127(5): 394-402.

27. Overdorp EJ, Kessels RP, Claassen JA, Oosterman JM The combined effect of neuropsychological and neuropathological deficits on instrumental activities of daily living in older adults: a systematic review. Neuropsychol Rev. 2016; 26(1): 92-106.

28. Roberts JL, Clare L Meta-representational awareness in mild cognitive impairment: an interpretative phenomenological analysis. Aging Ment Health. 2013; 17(3): 300-309.

29. Berg AI, Wallin A, Nordlund A, Johansson B Living with stable MCI: Experiences among 17 individuals evaluated at a memory clinic. Aging Ment Health. 2013; 17(3): 293-399.

30. Parikh PK, Troyer AK, Maione AM, Murphy KJ The impact of memory change on daily life in normal aging and mild cognitive impairment. Gerontologist. 2016; 56(5): 877-885.

31. Marioni RE, Proust-Lima C, Amieva H, et al. Social activity, cognitive decline and dementia risk: a 20 -year prospective cohort study. BMC Public Health. 2015; 15(1): 1089.

32. Norton S, Matthews FE, Barnes DE, Yaffe K, Brayne C Potential for primary prevention of Alzheimer's disease: an analysis of population-based data. Lancet Neurol. 2014; 13(8): 788-794.

33. Van der Mussele S, Fransen F, et al. Depression in mild cognitive impairment is associated with progression to Alzheimer's disease: a longitudinal study. J Alzheimers Dis. 2014; 42(4): 12391250 .

34. Stephan BC, Hunter S, Harris D, et al. The neuropathological profile of mild cognitive impairment (MCI): a systematic review. Mol Psychiatry. 2012; 17(11): 1056-1076.

35. Fennema-Notestine C, McEvoy LK, Hagler DJ Jr, Jacobson MW, Dale AM Structural neuroimaging in the detection and prognosis of pre-clinical and early AD. Behav Neurol. 2009; 21(1): 3-12.

36. Kim SH, Seo SW, Yoon DS, et al. Comparison of neuropsychological and FDG-PET findings between early- versus late-onset mild cognitive impairment: a five-year longitudinal study. Dement Geriatr Cogn Disord. 2010; 29(3): 213-223.

37. Habert MO, Horn JF, Sarazin M, et al. Brain perfusion SPECT with an automated quantitative tool can identify prodromal Alzheimer's disease among patients with mild cognitive impairment. Neurobiol Aging. 2011; 32(1): 15-23.

38. Zhang S, Smailagic N, Noel-Storr AH, Takwoingi Y, McShane R, Feng J (11)C-PIB-PET for the early diagnosis of Alzheimer's disease dementia and other dementias in people with mild cognitive impairment (MCI). Cochrane Database Syst Rev. 2014; (7): CD010386.

39. Ritchie C, Smailagic N, Noel-Storr AH, Ukoumunno O, Ladds EC, Martin S CSF tau and the CSF tau/ABeta ratio for the diagnosis of Alzheimer's disease dementia and other dementias in people with mild cognitive impairment (MCI) (Review). Cochrane Database Syst Rev. 2017; (3): CD010803.

40. Ritchie C, Smailagic N, Noel-Storr AH, et al. Plasma and cerebrospinal fluid amyloid beta for the diagnosis of Alzheimer's disease dementia and other dementias in people with mild cognitive impairment (MCI). Cochrane Database Syst Rev. 2014; 10(6): CD008782.

41. Qian J, Wolters FJ, Beiser A, et al. APOE-related risk of mild cognitive impairment and dementia for prevention trials: an analysis of four cohorts. PLoS Med. 2017; 14(3): e1002254.

42. Berchtold NC, Sabbagh MN, Beach TG, Kim RC, Cribbs DH, Cotman CW Brain gene expression patterns differentiate mild 
cognitive impairment from normal aged and Alzheimer's disease. Neurobiol Aging. 2014; 35(9): 1961-1972.

43. Folstein MF, Folstein SE, McHugh PR 'Mini-mental state'. A practical method for grading the cognitive state of patients for the clinician. J Psychiatr Res. 1975; 12(3): 189-198.

44. Dickerson BC, Salat DH, Greve DN, et al. Increased hippocampal activation in mild cognitive impairment compared to normal aging and AD. Neurology. 2005; 65(3): 404-411.

45. Putcha D, Brickhouse M, O'Keefe K, et al. Hippocampal hyperactivation associated with cortical thinning in Alzheimer's disease signature regions in non-demented elderly adults. J Neurosci. 2011; 31(48): 17680-17688.

46. Jorm AF Is depression a risk factor for dementia or cognitive decline? A review. Gerontology. 2000; 46(4): 219-227.

47. Slavin MJ, Brodaty H, Kochan NA, et al. Prevalence and predictors of 'subjective cognitive complaints' in the Sydney Memory and Ageing Study. Am J Geriatr Psychiatry. 2010; 18(8): 701-710.

48. Ownby RL, Crocco E, Acevedo A, John V, Loewenstein D Depression and risk for Alzheimer disease: systematic review, meta-analysis, and meta-regression analysis. Arch Gen Psychiatry. 2006 ; 63(5): 530-538.

49. Kuchibhatla MN, Fillenbaum GG, Hybels CF, Blazer DG (2012). Trajectory classes of depressive symptoms in a community sample of older adults. Acta Psychiatr Scand. 2012; 125(6): 492-501.

50. Buldú JM, Bajo R, Maestú F, et al. Reorganization of functional networks in mild cognitive impairment. PLoS One. 2011; 6(5): e19584.

51. Seo EH, Lee DY, Lee JM, et al. Whole-brain functional networks in cognitively normal, mild cognitive impairment, and Alzheimer's disease. PLoS One. 2013; 8(1): e53922.

52. McBride J, Zhao X, Munro N, et al. Spectral and complexity analysis of scalp EEG characteristics for mild cognitive impairment and early Alzheimer's disease. Comput Methods Programs Biomed. 2014; 114(2): 153-163.

53. Bruña R, Poza J, Gómez C, García M, Fernández A, Hornero R Analysis of spontaneous MEG activity in mild cognitive impairment and Alzheimer's disease using spectral entropies and statistical complexity measures. J Neural Eng. 2010; 9(3): 036007.

54. McIntosh AR, Kovacevic N, Itier RJ Increased brain signal variability accompanies lower behavioral variability in development. PLoS Comput Biol. 2008; 4(7): e1000106.

55. Dauwels J, Srinivasan K, Ramasubba Reddy M, et al. Slowing and loss of complexity in Alzheimer's EEG: two sides of the same coin? Int J Alzheimers Dis. 2011; 2011: 539621.

56. Braak H, Braak E Staging of Alzheimer's disease-related neurofibrillary changes. Neurobiol Aging. 1995; 16(3): 271-278.

57. Taylor KI, Probst A Anatomical localization of the transentorhinal regions of the perirhinal cortex. Neurobiol Aging. 2008; 29(10): 1591-1596.

58. Zhou M, Zhang F, Zhao L, Qian J, Dong C Entorhinal cortex: a good biomarker for mild cognitive impairment and mild Alzheimer's disease. Rev Neurosci. 2016; 27(2): 185-195.

59. Leal SL, Yassa MA Perturbations of neural circuitry in aging, mild cognitive impairment, and Alzheimer's disease. Ageing Res Rev. 2013; 12(3): 823-831.

60. Nasreddine ZS, Phillips NA, Bédirian V, et al. The Montreal Cognitive Assessment, MoCA: a brief screening tool for mild cognitive impairment. J Am Geriatr Soc. 2005; 53(4): 695-699.

61. Olsen RK, Yeung L-K, Noly-Gandon A, et al. Human anterolateral entorhinal cortex volumes are associated with cognitive decline in aging prior to clinical diagnosis. Neurobiol Aging. 2017; 57: 195-205.

62. Wolk DA, Das SR, Mueller SG, Weiner MW, Yushkevich PA Medial temporal lobe regional morphometry using high resolution in Alzheimer's disease. Neurobiol Aging. 2017; 49: 204-213.

63. Sone D, Imabayashi E, Maikusa N, et al. Regional tau deposition and subregion atrophy of medial temporal structures in early
Alzheimer's disease: a combined positron emission tomography/ magnetic resonance imaging study. Alzheimers Dement (Amst). 2017; 9: 35-40.

64. Herukka S-K, Hviid Simonsen A, Andreasen N, et al. Recommendations for cerebrospinal fluid Alzheimer's disease biomarkers in the diagnostic evaluation of mild cognitive impairment. Alzheimers Dement. 2017; 13(3): 285-295.

65. Nakamura A, Kaneko N, Villemangne VL, et al. High performance plasma amyloid- $\beta$ biomarkers for Alzheimer's disease. Nature 2018; 554: 249-254.

66. Julayanont P, Brousseau M, Chertkow H, Phillips N, Nasreddine ZS Montreal Cognitive Assessment memory index score (MoCA-MIS) as a predictor of conversion from mild cognitive impairment to Alzheimer's disease. J Am Geriatr Soc. 2014; 62(4): 679-684.

67. Brooks BL, Iverson GL, Holdnack JA, Feldman HH Potential for misclassification of mild cognitive impairment: a study of memory scores on the Wechsler Memory Scale-III in healthy older adults. J Int Neuropsychol Soc. 2008; 14(3): 463-478.

68. Mistridis P, Egli SC, Iverson GL, et al. Considering the base rates of low performance in cognitively healthy older adults improves the accuracy to identify neurocognitive impairment with the Consortium to Establish a Registry for Alzheimer's DiseaseNeuropsychological Assessment Battery (CERAB-NAB). Eur Arch Psychiatr Clin Neurosci. 2015; 265(5): 407-417.

69. Malek-Ahmadi M Reversion from mild cognitive impairment to normal cognition: a meta-analysis. Alzheimer Dis Assoc Disord. 2016; 30(4): 324-330.

70. Lenehan ME, Klekociuk SZ, Summers MJ Absence of a relationship between subjective memory complaint and objective memory impairment in mild cognitive impairment (MCI): is it time to abandon subjective memory complaint as a MCI diagnostic criterion? Int Psychogeriatr. 2012; 24(9): 1505-1514.

71. Roberts JL, Clare L, Woods RT Subjective memory complaints and awareness of memory functioning in mild cognitive impairment: a systematic review. Dement Geriatr Cogn Disord. 2009; 28(2): 95-109.

72. Edmonds EC, Delano-Woods L, Galasko DR, Salmon DP, Bondi MW Subjective cognitive complaints contribute to the misdiagnosis of mild cognitive impairment. J Int Neuropsychol Soc. 2014; 20(8): 836-847.

73. Barense MD, Gaffan D, Graham KS The human medial temporal lobe processes online representations of complex objects. Neuropsychologia. 2007; 45(13): 2963-2974.

74. Kivisaari SL, Tyler LK, Monsch AU, Taylor KI Medial perirhinal cortex disambiguates confusable objects. Brain. 2012; 135(12): 3757-3769.

75. Lee AC, Bandelow S, Schwarzbauer C, Henson RN, Graham KS Perirhinal cortex activity during visual object discrimination: an event-related fMRI study. Neuroimage. 2006; 33(1): 362-273.

76. Ranganath C, Ritchey M Two cortical systems for memory-guided behavior. Nat Rev Neurosci. 2012; 13(10): 713-726.

77. Ahmed S, Mitchell J, Arnold R, Dawson K, Nestor PJ, Hodges JR Memory complaints in mild cognitive impairment, worried well, and semantic dementia patients. Alzheimers Dis Assoc Disord. 2008; 22(3): 227-235.

78. Farias ST, Mungas D, Reed BR, Harvey D, Cahn-Weiner D, DeCarli C MCI is associated with deficits in everyday functioning. Alzheimer Dis Assoc Disord. 2006; 20(4): 217-223.

79. Bowles B, Crupi C, Pigott S, et al. Double dissociation of selective recollection and familiarity impairments following two different surgical treatments for temporal-lobe epilepsy. Neuropsychologia. $2010 ;$ 48(9): 2640-2647.

80. Bowles B, Duke D, Rosenbaum RS, McRae K, Köhler S Impaired assessment of cumulative lifetime familiarity for object concepts after left anterior temporal-lobe resection that includes perirhinal 
cortex but spares the hippocampus. Neuropsychologia. 2016; 90: 170-179.

81. Martin CB, Bowles B, Mirsattari SM, Köhler S Selective familiarity deficits after left anterior temporal-lobe removal with hippocampal sparing are material specific. Neuropsychologia. 2011; 49(7): 1870-1878.

82. Fidalgo CO, Changoor AT, Page-Gould E, Lee ACH, Barense MD Early cognitive decline in older adults better predicts object than scene recognition performance. Hippocampus. 2016; 26(12): 1579-1592.

83. Alegret M, Boada-Rovira M, Vinyes-Junqué G, et al. Detection of visuoperceptual deficits in preclinical and mild Alzheimer's disease. J Clin Exp Neuropsychol. 2009; 31(7): 860-867.

84. Anderson ND, Ebert PL, Jennings JM, Grady CL, Cabeza R, Graham SJ Recollection- and familiarity-based memory in healthy aging and amnestic mild cognitive impairment. Neuropsychol. 2008; 22(2): 177-187.

85. Hudon C, Belleville S, Gauthier S The assessment of recognition memory using the Remember/Know procedure in amnestic mild cognitive impairment and probably Alzheimer's disease. Brain Cogn. 2009; 70(1): 171-179.

86. Ally BA, Gold CA, Budson AE An evaluation of recollection and familiarity in Alzheimer's disease and mild cognitive impairment. Brain Cogn. 2009; 69(3): 504-513.

87. Wolk DA, Mancuso L, Kliot D, Arnold S, Dickerson BC Familiarity-based memory as an early cognitive marker of preclinical and prodromal AD. Neuropsychologia. 2013; 51(6): 1094-1102.

88. Schoemaker D, Poirier J, Escobar S, Gauthier S, Pruessner J Selective familiarity deficits in otherwise cognitively intact aging individuals with genetic risk for Alzheimer disease. Alzheimers Dement (Amst) 2016; 2: 132-139.

89. Roberts JS, Karlawish JH, Uhlmann WR, Petersen RC, Green RC Mild cognitive impairment in clinical care: A survey of American Academy of Neurology members. Neurology. 2010; 75(5): 425-431.

90 Stern Y. Cognitive reserve in aging and Alzheimer's disease. Lancet Neurol. 2012; 11(11): 1006-1012.

91. Ströhle A, Schmidt DK, Schultz F, et al. Drug and exercise treatment of Alzheimer disease and mild cognitive impairment: a systematic review and meta-analysis of effects on cognition in randomized controlled trials. Am J Geriatr Psychiatry. 2015; 23 (12): 1234-1249.

92. Baker LD, Frank LL, Foster-Schubert K, et al. Effects of aerobic exercise on mild cognitive impairment: a controlled trial. Arch Neurol. 2010; 67(1): 71-79.

93. Colcombe $\mathrm{S}$, Kramer AF Fitness effects on the cognitive function of older adults: a meta-analytic study. Psychol Sci. 2003; 14(2): 125-130.

94. Middleton L, Kirkland S, Rockwood K Prevention of CIND by physical activity: different impact on VCI-ND compared with MCI. J Neurol Sci. 2008; 269(1-2): 80-84.

95. Singh B, Parsaik AK, Mielke MM, et al. Association of Mediterranean diet with mild cognitive impairment and Alzheimer's disease: a systematic review and meta-analysis. J Alzheimers Dis. 2014; 39(2): 271-282.

96. Bayer-Carter JL, Green PS, Montine TJ, et al. Diet interventions and cerebrospinal fluid markers in amnestic mild cognitive impairment. Arch Neurol. 2011; 68(6): 743-752.
97. Baker LD, Bayer-Carter JL, Skinner J, et al. High-intensity physical activity modulates diet effects on cerebrospinal amyloid- $\beta$ levels in normal aging and mild cognitive impairment. J Alzheimers Dis. 2012; 28(1): 137-146.

98. Belleville S, Gilbert B, Fontaine F, Gagnon L, Ménard E, Gauthier $\mathrm{S}$ Improvement of episodic memory in persons with mild cognitive impairment and healthy older adults: evidence from a cognitive intervention program. Dement Geriatr Cogn Disord. 2006; 22(5-6): 486-489.

99. Belleville S, Hudon C, Bier N, et al. MEMO + : Efficacy, durability, and effect of cognitive training and psychosocial intervention in individuals with mild cognitive impairment. J Am Geriatr Soc. 2018 ; 66(4): 655-663.

100. Jeong JH, Na HR, Choi SH, et al. Group- and home-based cognitive interventions for patients with mild cognitive impairment: a randomized controlled trial. Psychother Psychosom. 2016; 85(4): 198-207.

101. Schmitter-Edgecombe M, Dyck DG Cognitive rehabilitation multifamily group intervention for individuals with mild cognitive impairment and their care-partners. J Int Neuropsychol Soc. 2014; 20(9): 897-908.

102. Troyer AK, Murphy KM, Anderson ND, Moscovitch M, Craik FI Changing everyday memory behavior in amnestic mild cognitive impairment: a randomised controlled trial. Neuropsychol Rehabil. 2008; 18(1): 65-88.

103. Anderson ND, Murphy KM, Troyer AK Living with Mild Cognitive Impairment: A Guide to Maximizing Brain Health and Reducing Risk of Dementia. New York, NY: Oxford University Press; 2012.

104. Callahan BL, Anderson ND Effect of conceptual and lexical errorless learning versus trial-and-error learning in amnestic mild cognitive impairment. Neuropsychol Rehabil. In press. doi: 10.1080/09602011.2017.1361843

105. Lubinsky T, Rich JB, Anderson ND Errorless learning and elaborative self-generation in healthy older adults and individuals with amnestic mild cognitive impairment: mnemonic benefits and mechanisms. J Int Neuropsychol Soc. 2009; 15(5): 704-716.

106. Roberts JL, Anderson ND, Guild E, Cyr A-A, Jones RSP, Clare L The benefits of errorless learning for people with amnestic mild cognitive impairment. Neuropsychol Rehabil. 2018; 28(6): 984-996.

107. Carretti B, Borella E, Fostinelli S, Zavagnin M Benefits of training working memory in amnestic mild cognitive impairment: specific and transfer effects. Int Psychogeriatr. 2013; 25(4): 617-626.

108. Vermeij A, Claassen JA, Dautzenberg PL, Kessels RP Transfer and maintenance effects of online working-memory training in normal ageing and mild cognitive impairment. Neuropsychol Rehabil. 2016; 26(5-6): 783-809.

109. Finn M, McDonald S Repetition-lag training to improve recollection memory in older people with amnestic mild cognitive impairment: a randomized controlled trail. Aging Neuropsychol Cogn. 2015; 22(2): 244-258.

110. Jennings JM, Lopina EC, Dagenbach D Improving memory and executive function in older adults with memory impairments through repetition-lag training. In: Lindsay DS, Kelley CM, Yonelinas AP, Roediger III HL, ed. Remembering: Attributions, Processes, and Control in Human Memory. Essays in Honor of Larry Jacoby. New York, NY: Psychology Press; 2015: 252-268.

111. Corlan AD Medline trend: automated yearly statistics of PubMed results for any query. 2004. http://dan.corlan.net/medline-trend. html. Accessed February 3, 2018. 